\title{
Editorial
}

\section{Essential service delivery in reproductive, maternal and child health services during the pandemic}

\section{Chithramalee De Silva \& Hemali Jayakody*}

Family Health Bureau, Ministry of Health, Sri Lanka

*Correspondence: gayathri_hj@yahoo.com

DOI: https://doi.org/10.4038/jccpsl.v26i4.8401

Received on 23 December 2020

Accepted on 30 December 2020

\section{Introduction}

Any pandemic results in more indirect damage to health outcomes of population than the direct effects. Reproductive, maternal, newborn, child, adolescent and youth health services (RMNCAYH services) are vulnerable to health system changes which happen in a pandemic. Predicted global excess mortality for children was 253,000 and excess maternal mortality was 12,190 . Both will be due to the disruption of health services during the pandemic (1). Impact on RMNCAYH services will disproportionately affect low- and middle-income countries (LMIC) whose buying capacity is low and lacks infrastructure in health service delivery. Diversion of resources to manage COVID-19 impacts on care provision in LMICs than in high-income countries.

Implications of the pandemic on sexual, reproductive and child health are multiple. Diversion of resources to care for infected patients, reduction in health facilities for maternity care, lack of health staff and disruptions to the medical supplies chain are some of the effects on the health system affecting women and children (2). Health facilities and health staff could be re-assigned to cater to the needs of an increasing number of COVID-19 patients. Diversion of health workforce disrupts routine essential services (3). Health workforce could get infected from the virus, may become carers of family members or will self- https://orcid.org/0000-0002-0444-2530

isolate after an exposure. Health care campaigns and community engagement programs could not be continued in the pre-pandemic calibre. Complicating the scenario further, social determinants of health such as social support systems, income, transport and access have got affected during the COVID-19 pandemic (1). Closure of schools, formal and informal education centres results in decreasing the opportunities for interaction for targeted health interventions such as the school health programme.

Health seeking behaviour of people got affected due to fear of accessing care (4). Extensive lockdowns resulted in lack of transport which in turn affected limited access to health services. Within the family, lack of social contacts and income leads to lack of access to food and other supplies. It could result in mental stress which contributes to gender-based violence, poor mental wellbeing and an increased tendency to substance abuse (4). Disruption to education and lack of access to digital services leads to long-term negative impact on the education of children. The pandemic and resulting negative impact on education and social opportunities create stress and mental health issues among children and adolescents.

The RMNCAYH programme in Sri Lanka suffered to some extent due to the pandemic. Its main service delivery platforms - home visits, clinics and hospitals 
- got affected due to public health measures to contain COVID-19. However, most essential health services were provided throughout the pandemic. Antenatal care, postpartum, and newborn care were continued. Institutional deliveries and skilled attendance at birth remained unchanged. However, analysis of maternal deaths during the first wave in the country revealed poor quality of care.

There were many mitigation strategies used to maintain essential services in RMNCAYH throughout the pandemic in Sri Lanka. All mitigation strategies were aimed to ensure the accessibility and availability of essential health services when and where women needed them.

\section{Key strategies used to maintain essential health services}

Prioritization of health service delivery was the strategy used in the initial phase of the pandemic. It coincided with the social distancing measures and limitations in people movement. Core maternity services such as antenatal, postnatal and newborn care continued, whereas immunization and anthropometric assessment of children were temporarily halted. Many services for reproductiveage females except for family planning were not available in high-risk areas. COVID-19 is a prolonged pandemic. All these services are part of the comprehensive package to reduce maternal and child morbidity and mortality. However, the impact of lack of services will be evident in the long run.

A series of guidelines were issued for service providers in organizing care during the pandemic. The guidance was issued according to changing public health policies to manage the pandemic and changing demands on the public health staff. Health services adapted to responding to various circulars and guidelines caring for COVID-19 patients. Guideline dissemination utilized non-traditional methods such as emails, websites and social media platforms. Establishing protocols and guidance is an effective way of handling a disaster. Implementation of guidelines was hampered due to resource constraints and poor reach to the target group.
Gathering the public for regular health education sessions was not possible. It was replaced with sharing health messages across mass media, websites and social media. It enabled the national level experts to get in contact with the service users and provide direct guidance. However, the reach of the messages was highly variable. When addressing the public in general, precise advice could not be provided.

Telehealth was a significant addition to care provision during the pandemic. Public health midwives were instructed to contact clients over the phone, assess their risk and get them to a clinic or a hospital if necessary. Lack of formal training on providing an effective over-the-phone consultation or advice was a drawback, which needs to be addressed in near future. Many helplines were also included in the response when providing such services, including a dedicated helpline for survivors of gender-based violence.

Ensuring the safety of health services was imperative to gain the confidence of clients. The public will be confident to visit hospitals and clinics whenever necessary. Effective triage system to identify women and children with respiratory symptoms, provision of handwashing facility and use of personal protective equipment by health care workers were some of the measures taken to prevent transmission of the virus within the clinic or during inward care. Many measures were taken to enhance the use of personal protective equipment by the field health teams when providing domiciliary and field clinic services. Nonadherence to control measures consistently was a problem. Frequent audits and supervisions are required to ensure that a guideline is followed.

\section{Conclusion}

The unique and novel situation created by COVID-19 in the country has been a learning experience for all the sectors involved. Setbacks will be expected to happen even in the well-established maternal and child healthcare systems and wellbeing of the families in the years to come. It may cause difficulties in reaching national health targets and Sustainable Development Goals. Therefore, all those who are involved in the provision of $\mathrm{MCH}$ care in the country 
must be ready to sustain these services through continuous advocacy, capacity building and research. The gaps in service provision need to be identified and strengthen with effective mitigation strategies.

\section{References}

1. Roberton T, Carter ED, Chou VB, Stegmuller AR, Jackson BD, Tam Y, et al. Early estimates of the indirect effects of the COVID-19 pandemic on maternal and child mortality in low-income and middle-income countries: a modelling study. The Lancet Global Health 2020; 8(7): e901-e8.
2. Hussein J. COVID-19: what implications for sexual and reproductive health and rights globally? Sexual and Reproductive Health Matters 2020; 28(1): 1746065.

3. World Health Organization Regional Office for South-East Asia. Continuing essential sexual, reproductive, maternal, neonatal, child and adolescent health services during COVID-19 pandemic: practical considerations. New Delhi: WHO-SEARO, 04/05/2020.

4. Douglas M, Katikireddi SV, Taulbut M, McKee M, McCartney G. Mitigating the wider health effects of covid-19 pandemic response. BMJ 2020; 369:m1557. 